\title{
Monitoring oxidative stress during chronic obstructive pulmonary disease exacerbations using malondialdehyde
}

\author{
BalAZS ANTUS, ${ }^{1,2}$ GABRIELLA HARNASI, ${ }^{3}$ ORSOlYa DROZDOVSZKY, ${ }^{2}$ IMRE BARTA ${ }^{1}$ \\ Departments of ${ }^{1}$ Pulmonology, ${ }^{2}$ Pathophysiology and ${ }^{3}$ Pathology, National Koranyi Institute of TB and Pulmonology, \\ Budapest, Hungary
}

\begin{abstract}
Background and objective: Oxidative stress plays an important role in the pathogenesis of chronic obstructive pulmonary disease (COPD). In this longitudinal study changes in the level of malondialdehyde (MDA), an end product of polyunsaturated fatty acid peroxidation, were investigated in the airways of patients with acute exacerbation of COPD (AECOPD).

Methods: Levels of MDA were measured in sputum and exhaled breath condensate (EBC) of 34 COPD patients at the time of hospital admission due to an acute exacerbation of the disease, and again following treatment at the time of hospital discharge. MDA was also assessed in 21 stable patients with COPD and 20 healthy controls. Measurements were performed using high-performance liquid chromatography.

Results: Sputum MDA levels were significantly increased in AECOPD $(220.0 \pm 17.5 \mathrm{nmol} / \mathrm{L})$ compared with stable disease $(144.6 \pm 14.3 \mathrm{nmol} / \mathrm{L}, P<0.01)$ and healthy controls $(85.9 \pm 11.3 \mathrm{nmol} / \mathrm{L}, \quad P<0.001)$. MDA levels decreased after treatment $(190.7 \pm$ $16.3 \mathrm{nmol} / \mathrm{L}, P<0.05)$. In contrast to sputum, EBC MDA levels were comparable between controls, stable COPD patients and AECOPD patients (73.1 \pm $5.1 \mathrm{nmol} / \mathrm{L}, 96.1 \pm 11.6 \mathrm{nmol} / \mathrm{L}$ and $93.3 \pm 7.6 \mathrm{nmol} / \mathrm{L}$, $P=$ NS). Measurement of MDA had good repeatability in both sputum and EBC, but the between-day variability was considerably higher in EBC. Sputum induction did not influence MDA levels.

Conclusions: MDA in sputum, but not in EBC, appears to be a useful marker for monitoring exacerbation-associated oxidative stress in AECOPD.
\end{abstract}

Key words: chronic obstructive pulmonary disease, exhaled breath condensate, oxidative stress, repeatability, sputum.

Abbreviations: AECOPD, acute exacerbation of chronic obstructive pulmonary disease; COPD, chronic obstructive pulmonary disease; $\mathrm{CR}$, coefficient of repeatability; $\mathrm{CV}$, coefficient of variation; EBC, exhaled breath condensate; FENO, fractional

Correspondence: Balazs Antus, Department of Pathophysiology, National Koranyi Institute of TB and Pulmonology, Piheno ut 1, H-1121 Budapest, Hungary. Email: antusb@hotmail.com

Received 15 February 2013; invited to revise 1 April 2013, 13 May 2013, 10 June 2013; revised 16 April 2013, 5 June 2013, 13 June 2013; accepted 16 June 2013 (Associate Editor: Paul Thomas).

\section{SUMMARY AT A GLANCE}

Malondialdehyde, an established by-product of lipid peroxidation, can be precisely measured in the sputum and is a useful marker to monitor exacerbation-associated oxidative stress in patients with COPD.

exhaled nitric oxide; $\mathrm{FEV}_{1}$, forced expiratory volume in one second; FVC, forced vital capacity; HPLC, high-performance liquid chromatography; MDA, malondialdehyde; NS, nonsignificant; $\mathrm{PaCO}_{2}$, arterial carbon dioxide tension; $\mathrm{PaO}_{2}$, arterial oxygen tension; WBC, white blood cell.

\section{INTRODUCTION}

Oxidative stress is thought to play a pivotal role in the pathogenesis of chronic obstructive pulmonary disease (COPD), particularly during acute exacerbations of COPD (AECOPD) ${ }^{1-3}$ Several processes lead to oxidative stress-related tissue damage, among them lipid peroxidation, in which oxidation of cell membrane phospholipids results in the formation of various lipid hydroperoxides and aldehydic products. $^{2}$ Among these molecules, malondialdehyde (MDA), a by-product of polyunsaturated fatty acid peroxidation, may be a reliable marker of oxidative stress in various diseases. ${ }^{4}$

Sputum induction and exhaled breath condensate (EBC) collection allow sampling of the airways in a non-invasive fashion. Sputum and EBC have been widely used to assess airway inflammation and oxidative stress in COPD. ${ }^{5}$ Both methods offer a unique opportunity to identify pulmonary biomarkers of potential clinical utility in the management of COPD. ${ }^{6}$

There is evidence that MDA can be measured accurately in EBC using high-performance liquid chromatography (HPLC). ${ }^{7}$ Using this technique it was recently demonstrated that MDA levels in $\mathrm{EBC}^{8,9}$ and induced sputum $^{10}$ are elevated in stable COPD compared with levels in healthy controls. It should be noted, however, that in some of these trials ${ }^{9,10}$ the group with COPD included current smokers. The 


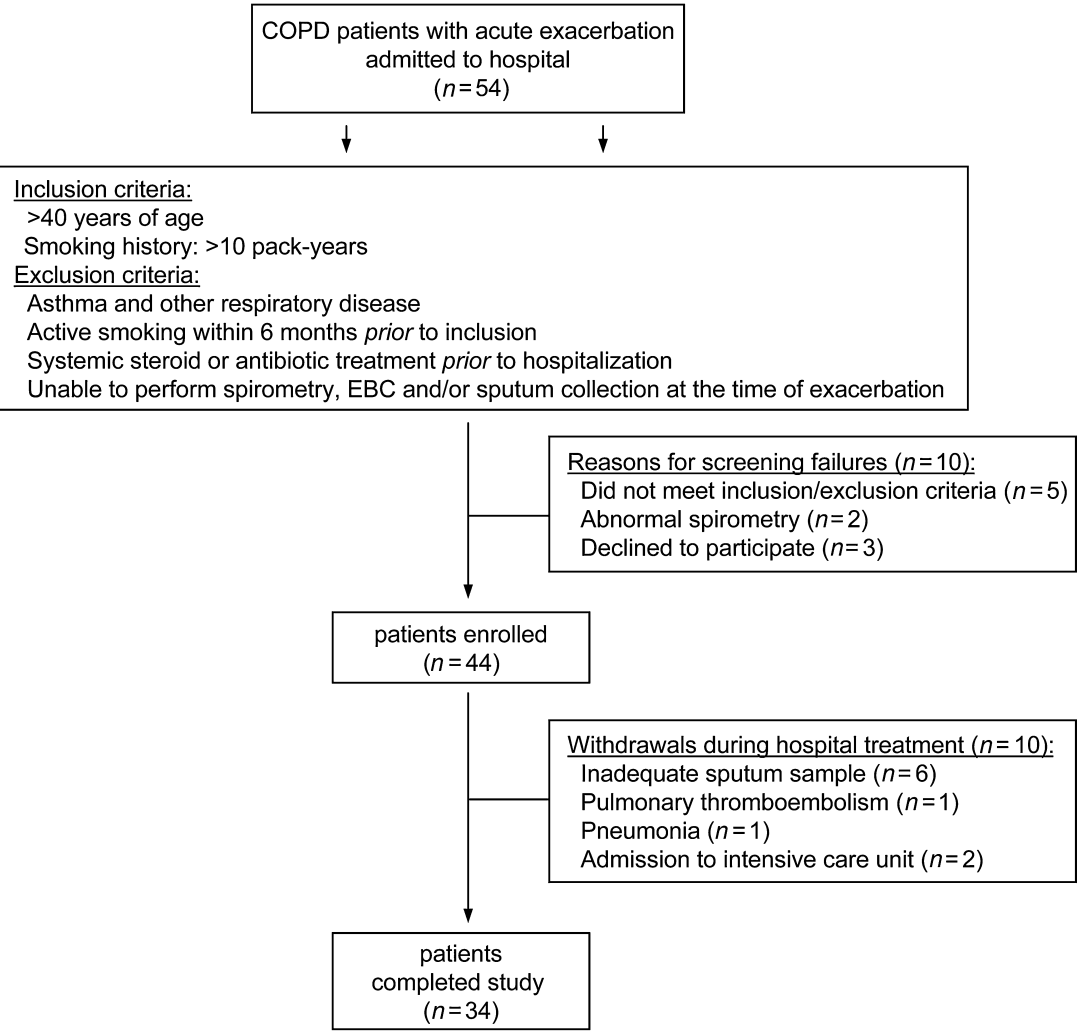

inclusion of smokers potentially affected the overall outcome of the study, as it has been shown, at least in EBC, that smoking alone increases MDA levels. ${ }^{8}$

The above-mentioned data suggest that MDA measurements can discriminate between persons with COPD and healthy subjects. However, the clinical utility of the test for monitoring oxidative stress during AECOPD is unclear. Moreover, it is unknown if EBC or sputum has superior clinical utility in this context.

In this longitudinal study we assessed the levels of MDA in sputum and EBC of patients with AECOPD at onset and after treatment. For comparison, MDA levels in stable COPD and healthy controls were also determined.

\section{METHODS}

\section{Study subjects}

Patients hospitalized with AECOPD were recruited for the study. Inclusion and exclusion criteria for participation are summarized in Figure 1. Exacerbation was defined as increased dyspnoea, cough or sputum expectoration that led the subject to seek medical attention, as specified in international guidelines. ${ }^{11}$

Additionally, 21 clinically stable ex-smoker COPD patients and 20 healthy ex-smoker controls were enrolled in the study (Table 1). All patients were $>40$ years of age, had a smoking history of $>10$ pack-years, and also had documented airway obstruction, with forced expiratory volume in one second $\left(\mathrm{FEV}_{1}\right)<80 \%$ of predicted value and postbronchodilator $\mathrm{FEV}_{1} /$ forced vital capacity (FVC ratio) $<0.7$. Control subjects had normal lung function values and no history of acute or chronic respiratory diseases in the previous 4 weeks. The local ethics committee approved the research protocol, and all subjects gave written informed consent to participate in the study.

\section{Study design}

In patients with AECOPD, EBC and spontaneously expectorated sputum were collected and levels of fractional exhaled nitric oxide (FENO), blood gases and lung function parameters were measured on hospital admission and on the day of discharge. In stable COPD patients and healthy controls, EBC and induced sputum samples were collected during routine clinical visits. Lung function, blood gas parameters and FENO were determined, as previously described..$^{12}$

\section{EBC collection}

EBC was collected for a period of 10 min using an EcoScreen condenser (Jaeger, Hoechberg, Germany), as described previously. ${ }^{12}$ All samples were stored frozen at $-80^{\circ} \mathrm{C}$ before analysis.

\section{Sputum induction and processing}

In stable COPD patients sputum was induced by the inhalation of a hypertonic saline solution (4\% sodium chloride) delivered by an ultrasonic nebulizer (Ultra- 
Table 1 Demographic and clinical characteristics of study subjects

\begin{tabular}{|c|c|c|c|c|}
\hline & Healthy controls & Stable COPD & \multicolumn{2}{|c|}{ COPD exacerbation } \\
\hline Subjects, $n$ & 20 & 21 & \multicolumn{2}{|c|}{34} \\
\hline \multicolumn{5}{|l|}{ Sex, $n$} \\
\hline Male & 10 & 13 & \multicolumn{2}{|c|}{20} \\
\hline Female & 10 & 8 & \multicolumn{2}{|c|}{14} \\
\hline Age (years) & $61.8 \pm 1.6$ & $63.1 \pm 1.8$ & \multicolumn{2}{|c|}{$64.2 \pm 2.5$} \\
\hline Smoking (pack-years) & $51.6 \pm 5.2$ & $39.5 \pm 3.9$ & \multicolumn{2}{|c|}{$40.5 \pm 4.2$} \\
\hline \multicolumn{5}{|l|}{ GOLD stages, $n(\%)$} \\
\hline I & NA & 4 (19) & \multicolumn{2}{|c|}{$2(6)$} \\
\hline II & NA & $12(57)$ & \multicolumn{2}{|c|}{$10(29)$} \\
\hline III & NA & $5(24)$ & \multicolumn{2}{|c|}{$15(44)$} \\
\hline IV & NA & $0(0)$ & \multicolumn{2}{|c|}{$7(21)$} \\
\hline \multicolumn{3}{|l|}{ Pulmonary function } & At admission & After treatment \\
\hline FVC (L) & $3.92 \pm 0.20$ & $3.39 \pm 0.16$ & $1.96 \pm 0.11$ & $2.16 \pm 0.13^{\#}$ \\
\hline FVC (\% of predicted) & $104.2 \pm 2.7$ & $83.4 \pm 2.9$ & $72.1 \pm 3.3$ & $79.9 \pm 3.4^{\# \#}$ \\
\hline $\mathrm{FEV}_{1}(\mathrm{~L})$ & $2.93 \pm 0.14$ & $1.74 \pm 0.13$ & $0.94 \pm 0.07$ & $1.11 \pm 0.08^{\# \#}$ \\
\hline $\mathrm{FEV}_{1}(\%$ of predicted) & $111.3 \pm 3.6$ & $63.1 \pm 4.3^{* *}$ & $43.7 \pm 2.85$ & $51.9 \pm 3.10^{\# \#}$ \\
\hline $\mathrm{FEV}_{1} / \mathrm{FVC}(\%)$ & $77.0 \pm 1.4$ & $49.4 \pm 2.2^{* *}$ & $0.48 \pm 0.02$ & $0.52 \pm 0.02^{\#}$ \\
\hline \multicolumn{5}{|l|}{ Blood gases } \\
\hline $\mathrm{PaCO}_{2}(\mathrm{kPa})$ & $5.2 \pm 0.13$ & $5.2 \pm 0.12$ & $5.33 \pm 0.17$ & $5.64 \pm 0.21$ \\
\hline $\mathrm{PaO}_{2}(\mathrm{kPa})$ & $10.3 \pm 0.73$ & $8.4 \pm 0.22^{*}$ & $6.92 \pm 0.23$ & $7.57 \pm 0.20^{\#}$ \\
\hline FENO $(p p b)^{\dagger}$ & $7.1(5.9-9.9)$ & $8.7(6.03-11.4)$ & $12.7(6.18-28.7)$ & $10.2(6.55-17.3)^{\#}$ \\
\hline
\end{tabular}

Data are presented as mean \pm SEM unless stated otherwise.

${ }^{*} P<0.05$ vs controls; ${ }^{*} P<0.001$ vs controls; ${ }^{*} P<0.05$ vs admission; $\# P<0.001$ vs admission.

${ }^{\dagger}$ Median (interquartile ranges).

COPD, chronic obstructive pulmonary disease; FENO, fractional exhaled nitric oxide; FEV 1 , forced expiratory volume in one second; FVC, forced vital capacity; GOLD, Global Initiative for Chronic Obstructive Pulmonary Disease; $\mathrm{PaCO}_{2}$, arterial carbon dioxide tension;

$\mathrm{PaO}_{2}$, arterial oxygen tension; ppb, parts per billion.

Neb 2000, DeVilbiss Healthcare Ltd, Tipton, UK) with an output set at $1 \mathrm{~mL} / \mathrm{min}$.

Induced and spontaneous sputum samples were processed similarly within 120 min from collection, as previously described. ${ }^{13}$ Samples were homogenized in PBS containing $0.1 \%$ dithiothreitol (DTT). Investigating a subset of samples $(n=6)$ we found that DTT has no effect on MDA assay $(206.9 \pm 51.7 \mathrm{nmol} / \mathrm{L}$ with DTT vs $145.2 \pm 26.7 \mathrm{nmol} / \mathrm{L}$ without DTT, $P=$ NS). At least 400 inflammatory cells were counted for each cytospin slide. The number of inflammatory cells in sputum was recorded as a percentage of total viable non-squamous cells.

\section{Measurement of MDA}

MDA concentrations in EBC and sputum supernatant were measured by an isocratic HPLC system using MDA reagent kit (Chromsystems Ltd, Munich, Germany), according to the method described by Lärstad et al. ${ }^{7}$ Analysis was performed using a HPLC unit with fluorescence detector (Jasco, FP-2020 Plus; ABL\&E-Jasco Ltd, Budapest, Hungary) equipped with a MDA HPLC column (Chromsystems). Excitation and emission wavelengths were 515 and $553 \mathrm{~nm}$, respectively. The flow rate was $1.0 \mathrm{~mL} / \mathrm{min}$. The limit of quantification was $0.01 \mu \mathrm{mol} / \mathrm{L}$.

In order to compare MDA levels in induced and spontaneous sputum, a subgroup of stable COPD patients $(n=10)$ capable of expectorating sputum spontaneously were selected in a pilot study. MDA concentrations in induced $(135.7 \pm 9.7 \mathrm{nmol} / \mathrm{L})$ and spontaneous sputum samples $(148.1 \pm 7.3 \mathrm{nmol} / \mathrm{L})$ collected from the same subjects were comparable $(P=\mathrm{NS})$. The coefficient of repeatability $(\mathrm{CR})$ was $48.7 \mathrm{nmol} / \mathrm{L}$. The limits of agreement were -30.8 and 55.7 .

\section{Intra- and inter-assay repeatability of MDA measurements}

In order to estimate the intra-assay repeatability of MDA readings, a subset of processed and analysed EBC $(n=12)$ and sputum $(n=12)$ samples derived from six patients with stable COPD and six controls were assessed. These samples were stored at $4^{\circ} \mathrm{C}$ for two weeks and then remeasured by HPLC. For calculation of the inter-assay repeatability of MDA measurements, sputum and EBC samples collected from a subset of COPD patients $(n=12)$ were divided into two aliquots, which were then processed and measured separately.

\section{Between-day variability of MDA measurements} In order to assess the between-day variability of MDA measurements, EBC and spontaneous sputum collections from the same subjects were repeated in a subgroup of stable COPD patients $(n=12)$ on two consecutive days. 


\section{Statistical analysis}

Data are presented as mean \pm SEM or median with interquartile range as appropriate. MDA concentrations were compared using one-way ANOvA with Newman-Keuls test for multiple comparisons. Paired Student's $t$-test (parametric data) and the Wilcoxon signed-rank test (non-parametric data) were used to compare measurements at the time of hospital admission and measurements at discharge. Differences in baseline parameters between stable patients and controls were analysed by unpaired $t$-test or the Mann-Whitney test. Correlations were analysed by Pearson's method. The repeatability and variability of MDA measurements were assessed using the coefficient of variation (CV) and the Bland-Altman test. Additionally, the $\mathrm{CR}$ was estimated. ${ }^{14}$ Calculations were performed with GraphPad Prism 4.0 (GraphPad Software Inc., San Diego, CA, USA). A $P$-value $<0.05$ was considered significant.

\section{RESULTS}

Fifty-four patients with AECOPD were screened; 44 fulfilled inclusion criteria and agreed to participate (Fig. 1). During hospital treatment 10 patients were withdrawn. Demographic and clinical data of the 34 patients who completed the study are presented in Table 1.

\section{Clinical variables during treatment of exacerbations}

Exacerbations were treated with systemic glucocorticoids and short-acting bronchodilators (anticholinergics and/or $\beta_{2}$-agonists) in all cases. In addition, 73\% of the patients received long-acting $\beta_{2}$-agonists or anticholinergics. Antibiotics were given to 19 patients. The majority of patients $(89 \%)$ were treated with oxygen. The mean length of hospitalization was $10.9 \pm 1.5$ days. During the course of treatment/ recovery lung function variables and $\mathrm{PaO}_{2}$ increased, while sputum total cell counts and the number of neutrophils significantly decreased (Table 1 and Supporting Information Table S1).

\section{Intra-assay repeatability of MDA measurements}

MDA levels in sputum or EBC did not change after the processed samples had been kept at $4^{\circ} \mathrm{C}$ for 2 weeks $(P=\mathrm{NS}) \quad$ (Supporting Information Table S2). The mean CV for repeated measurements and the limits of agreement were similar between sputum and EBC. The CR in sputum and EBC were 25.7 and $18.5 \mathrm{nmol} / \mathrm{L}$, respectively.

\section{Inter-assay repeatability of MDA measurements}

MDA concentrations in two aliquots of the same sputum and EBC sample were similar $(P=\mathrm{NS})$ (Supporting Information Table S2). Again, the mean CV and the limits of agreement were comparable between sputum and EBC. The CR in sputum and EBC were 54.9 and $34.0 \mathrm{nmol} / \mathrm{L}$, respectively.

\section{Between-day variability of MDA measurements} Sputum MDA levels measured in samples collected on two consecutive days from the same patients were similar $(P=\mathrm{NS})$ (Supporting information Table S2). The mean difference between the two values was $15.5 \mathrm{nmol} / \mathrm{L}$. Although EBC MDA levels at the two assessment points were also comparable $(P=\mathrm{NS})$, the mean difference between the measurements increased up to $30.9 \mathrm{nmol} / \mathrm{L}$. Moreover, the mean CV for repeated measurements was significantly higher and the limits of agreement markedly greater in EBC as compared with sputum. The CR in sputum and EBC were 43.2 and $75.1 \mathrm{nmol} / \mathrm{L}$, respectively.

\section{MDA in sputum}

MDA levels in sputum were higher in stable COPD patients than in healthy controls (144.6 \pm $14.3 \mathrm{nmol} / \mathrm{L}$ vs. $85.9 \pm 11.3 \mathrm{nmol} / \mathrm{L}, P<0.05$; Fig. 2a). In patients with AECOPD, sputum MDA concentrations $(220.0 \pm 17.5 \mathrm{nmol} / \mathrm{L})$ were further increased compared with stable COPD patients $(P<0.01)$. MDA levels significantly decreased with treatment (190.7 $\pm 16.3 \mathrm{nmol} / \mathrm{L}, P<0.05)$.

To further explore the changes in MDA levels among patients with AECOPD, subjects were stratified by tertiles for increases in $\mathrm{FEV}_{1}\left(\Delta \mathrm{FEV}_{1}\right.$ and $\Delta \mathrm{FEV}_{1}$ $\%$ of predicted) post-treatment (Supporting Information Table S3 and Supporting Information Figure S1). Patients in the highest and middle tertiles had sputum MDA levels that were significantly decreased at discharge compared with those at admission $(P<0.05)$. By contrast, MDA concentrations did not change during treatment $(P=\mathrm{NS})$ in patients in the lowest tertile.

\section{MDA in EBC}

EBC MDA concentrations in controls (73.1 \pm $5.1 \mathrm{nmol} / \mathrm{L})$, stable COPD patients $(96.1 \pm 11.6 \mathrm{nmol} / \mathrm{L})$ and patients with AECOPD $(93.3 \pm 7.6 \mathrm{nmol} / \mathrm{L})$ were similar $(P=$ NS) (Fig. 2b), and treatment had no effect on EBC MDA levels either $(85.3 \pm 7.1 \mathrm{nmol} / \mathrm{L}$, $P=\mathrm{NS})$.

\section{Correlations between MDA and other variables}

No significant correlations were observed between sputum or EBC MDA values and FENO, lung function, blood gas parameters or sputum total and differential cell counts measured either in stable disease or at exacerbation (data not shown).

\section{DISCUSSION}

This study assessed oxidative stress in the airways of COPD patients by measuring MDA, an established marker of lipid peroxidation in laboratory diagnostics. Our results demonstrate that (i) MDA concentrations in sputum are elevated in stable COPD patients 


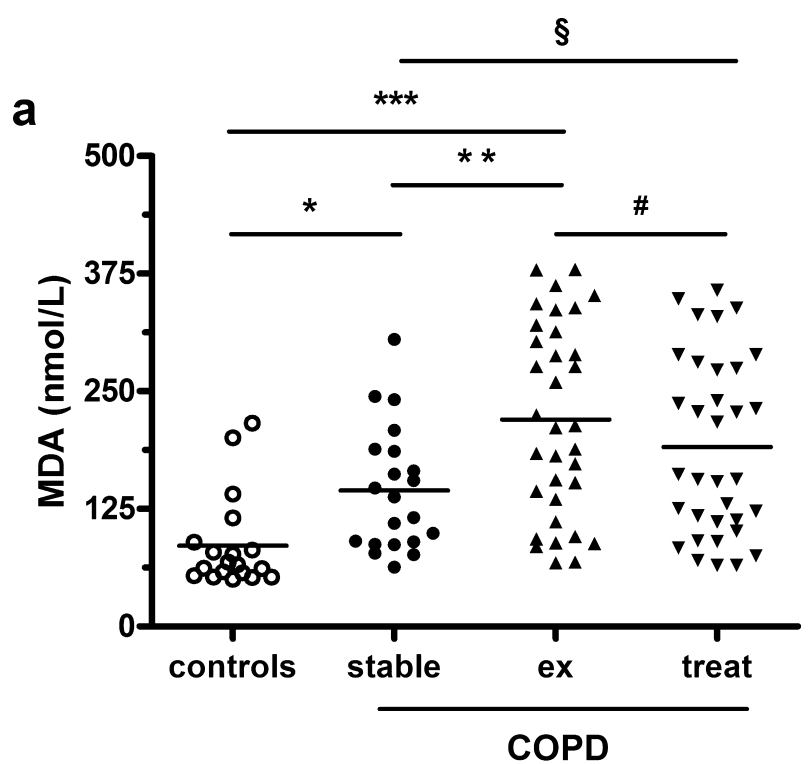

b

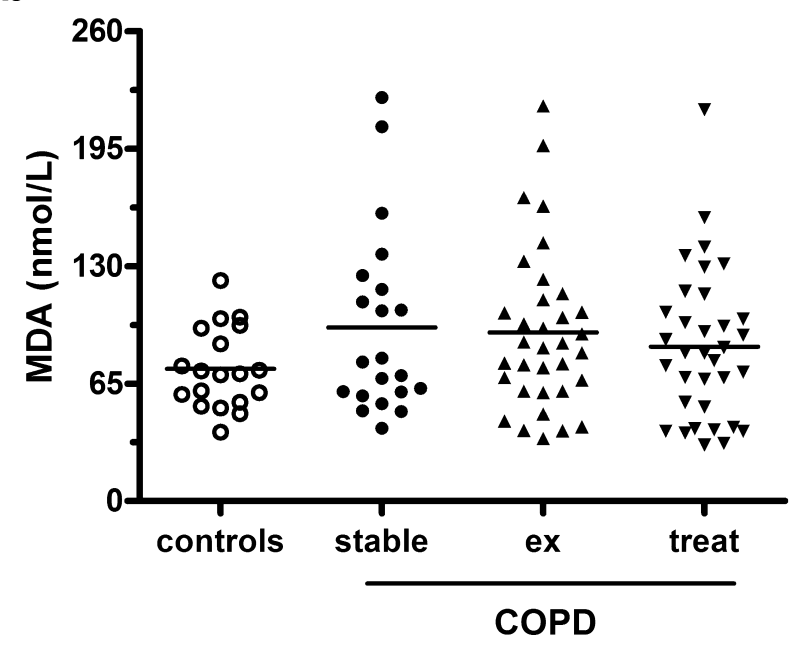

Figure 2 Malondialdehyde (MDA) concentrations in sputum (a) and in exhaled breath condensate (b) of healthy controls (controls), stable COPD patients (stable) and AECOPD patients at the time of acute exacerbation (ex) and after hospital treatment (treat). All subjects were ex-smokers. Horizontal bars represent mean values. ${ }^{*} P<0.05$ vs healthy controls; ${ }^{*} P<0.01$ vs stable COPD patients; ${ }^{* *} P<0.001$ vs healthy controls; ${ }^{*} P<0.05$ vs exacerbation; ${ }^{\S} P<0.05$ vs stable COPD patients.

compared with healthy controls; (ii) sputum MDA levels are further increased in AECOPD requiring hospitalization; and (iii) treatment of or recovery from exacerbation leads to a decrease in sputum MDA levels, primarily in those patients who have more pronounced improvement in airflow limitation posttreatment. Measurement of MDA in EBC, on the other hand, did not appear to reflect oxidative stress within the airways, possibly due to the high between-day variability for this marker in EBC.

Few longitudinal studies have investigated changes in MDA levels in the airways of patients with COPD experiencing an exacerbation. Corradi et al. have recently found that MDA decreases during corticosteroid treatment in children with an acute asthma attack. ${ }^{15}$ Here we demonstrate that in patients with AECOPD, levels of sputum MDA are increased compared with those of stable patients. This observation may reflect enhanced oxidant production during AECOPD.

Sputum MDA concentrations may have decreased after treatment. It is of interest that subgroup analysis found that the extent of changes in MDA level is different among patients with various functional improvements post-treatment. In patients who had pronounced increases in $\mathrm{FEV}_{1}$ after therapy (highest and middle tertiles) MDA levels were significantly decreased at discharge. However, in patients who experienced no or only a minor change in $\mathrm{FEV}_{1}$ (lowest tertile), MDA concentrations remained high.

Our observation that sputum MDA levels are elevated in stable COPD compared with healthy controls confirms the previous findings of Corradi et al. ${ }^{10}$ The ranges of MDA values reported in both studies are similar.

Patients with COPD and healthy controls could not be distinguished on the basis of MDA in EBC. AECOPD did not increase EBC MDA either. This finding may be due to the high between-day variability of MDA readings in EBC. In line with this view, considerable variability for other EBC biomarkers has been documented in several studies. ${ }^{16,17}$ Even the $\mathrm{pH}$, which is considered to be the most robust parameter of EBC, displays significant variation when assessed within a short period of time in the same subjects. ${ }^{18}$

The intra- and inter-assay repeatabilities of MDA measurements were comparable between EBC and sputum, indicating that our detection technique is reliable and has a similar threshold in sputum and EBC. MDA was measured by HPLC, a very sensitive method for analysing such compounds in aqueous matrices. ${ }^{19}$ After sample preparation the resulting fluorophore is highly specific and is detectable at very low levels using a fluorescence detector. This is of particular importance because EBC is extremely diluted, which generates a number of well-known methodological problems. Indeed, MDA was detectable in all EBC and sputum samples. Finally, it should be noted that our repeatability data, both $\mathrm{CV}$ and limits of agreement, are smaller than those reported for other EBC biomarkers measured by enzyme immunoassay, such as 8-isoprostane and leukotriene $\mathrm{B}_{4} \cdot{ }^{17}$

We are aware that sputum induction, as opposed to spontaneous sputum collection, is the method of choice in patients with mild-to-moderate COPD. ${ }^{20}$ However, as a large percentage $(>60 \%)$ of our recruits had severe or very severe COPD, spontaneous sputum collection, rather than sputum induction, was chosen in patients with AECOPD because of safety concerns. As the induction by itself had no effect on MDA readings, direct comparison of the two specimens was feasible.

In line with the findings of Corradi et al. ${ }^{10}$ we did not find correlations between sputum MDA levels and lung function parameters or inflammatory cell counts in our study. EBC MDA levels also did not correlate with clinical variables. Current data are equivocal as 
to whether such a relationship exists ${ }^{9}$ or not $^{10}$ in COPD and/or asthma.

In conclusion, this study suggests that assessment of sputum MDA, an established by-product of lipid peroxidation, reflects exacerbation-associated oxidative stress in patients with COPD. For EBC the high day-to-day variability may limit clinical applicability. The clinical utility of MDA measurements in the sputum should be investigated further to clarify its role in monitoring and to predict disease outcomes.

\section{Acknowledgements}

We thank M. Mikoss and J. Hernadi (National Koranyi Institute of TB and Pulmonology) for technical assistance in sputum processing and MDA measurements. We also thank Heather Shand and Brian Cook (Alfred Renyi Institute of Mathematics, Hungarian Academy of Sciences) for critical reading of the manuscript. The study was supported by the Hungarian National Scientific Foundation (OTKA K83338). Dr. B. Antus is a recipient of a Bolyai Janos Scholarship from the Hungarian Academy of Sciences.

\section{REFERENCES}

1 Tuder RM, Petrache I. Pathogenesis of chronic obstructive pulmonary disease. J. Clin. Invest. 2012; 122: 2749-55.

2 Rahman I, Adcock IM. Oxidative stress and redox regulation of lung inflammation in COPD. Eur. Respir. J. 2006; 28: 219-42.

3 Bathoorn E, Kerstjens H, Postma D et al. Airways inflammation and treatment during acute exacerbations of COPD. Int. J. Chron. Obstruct. Pulmon. Dis. 2008; 3: 217-29.

4 Rahman I, Biswas SK. Non-invasive biomarkers of oxidative stress: reproducibility and methodological issues. Redox. Rep. 2004; 9: 125-43.

5 Hillas G, Loukides S, Kostikas K et al. Biomarkers obtained by non-invasive methods in patients with COPD: where do we stand, what do we expect? Curr. Med. Chem. 2009; 16: 2824-38.

6 Barnes PJ, Chowdhury B, Kharitonov SA et al. Pulmonary biomarkers in chronic obstructive pulmonary disease. Am. J. Respir. Crit. Care Med. 2006; 174: 6-14.

7 Lärstad M, Ljungkvist G, Olin AC et al. Determination of malondialdehyde in breath condensate by high-performance liquid chromatography with fluorescence detection. J. Chromatogr. B Analyt. Technol. Biomed. Life Sci. 2002; 766: 107-14.

8 Corradi M, Rubinstein I, Andreoli R et al. Aldehydes in exhaled breath condensate of patients with chronic obstructive pulmonary disease. Am. J. Respir. Crit. Care Med. 2003; 167: 1380-6.

9 Bartoli ML, Novelli F, Costa F et al. Malondialdehyde in exhaled breath condensate as a marker of oxidative stress in different pulmonary diseases. Mediators Inflamm. 2011; 891752 1-7.

10 Corradi M, Pignatti P, Manini P et al. Comparison between exhaled and sputum oxidative stress biomarkers in chronic airway inflammation. Eur. Respir. J. 2004; 24: 1011-7.
11 Rabe KF, Hurd S, Anzueto A et al. Global strategy for the diagnosis, management, and prevention of chronic obstructive pulmonary disease: GOLD Executive Summary. Am. J. Respir. Crit. Care Med. 2007; 176: 532-55.

12 Antus B, Barta I, Kullmann T et al. Assessment of exhaled breath condensate $\mathrm{pH}$ in exacerbations of asthma and chronic obstructive pulmonary disease: a longitudinal study. Am. J. Respir. Crit. Care Med. 2010; 182: 1492-7.

13 Antus B, Barta I, Csiszer E et al. Exhaled breath condensate $\mathrm{pH}$ in patients with cystic fibrosis. Inflamm. Res. 2012; 61: 1141-7.

14 Bland JM, Altman DG. Statistical methods for assessing agreement between two methods of clinical measurement. Lancet 1986; 1: 307-10.

15 Corradi M, Folesani G, Andreoli R et al. Aldehydes and glutathione in exhaled breath condensate of children with asthma exacerbation. Am. J. Respir. Crit. Care Med. 2003; 167: 395-9.

16 Franklin P, Moeller A, Hall GL et al. Variability of nitric oxide metabolites in exhaled breath condensate. Respir. Med. 2006; 100: 123-9.

17 Borrill ZL, Starkey RC, Singh SD. Variability of exhaled breath condensate leukotriene B4 and 8-isoprostane in COPD patients. Int. J. Chron. Obstruct. Pulmon. Dis. 2007; 2: 71-6.

18 Kullmann T, Barta I, Antus B et al. Drinking influences exhaled breath condensate acidity. Lung 2008; 186: 263-8.

19 Giera M, Lingeman H, Niessen WM. Recent advancements in the LC- and GC-based analysis of malondialdehyde (MDA): a brief overview. Chromatographia 2012; 75: 433-40.

20 Bathoorn E, Liesker J, Postma D et al. Safety of sputum induction during exacerbations of COPD. Chest 2007; 131: 432-8.

\section{Supporting Information}

Additional Supporting Information may be found in the online version of this article at the publisher's web-site:

Figure $\mathbf{S} 1$ Individual changes in sputum malondialdehyde (MDA) values in patients with acute exacerbation of chronic obstructive pulmonary disease (AECOPD) stratified by tertiles for increases in forced expiratory volume in one second ( $\triangle \mathrm{FEV}{ }_{1} \%$ of predicted) post-treatment. Measurements were performed at the time of exacerbation (ex) and at discharge after hospital treatment (treat). Horizontal bars represent mean values. ${ }^{*} P<0.05$ vs. exacerbation.

Table S1 Total and differential sputum cell counts in study subjects.

Table S2 The intra- and inter-assay repeatability and the between-day variability of malondialdehyde (MDA) measurements in sputum and exhaled breath condensate (EBC) collected from a subset $(n=12)$ of stable COPD patients.

Table S3 Changes in sputum malondialdehyde (MDA) concentrations in patients with acute exacerbation of chronic obstructive pulmonary disease (AECOPD) as assessed by increases in FEV after treatment. 